\title{
Physics motivations and detector upgrades for the new era of the ATLAS experiment
}

\author{
Marisilvia Donadelli, on behalf of the ATLAS Collaboration ${ }^{a, *}$ \\ ${ }^{a}$ Instituto de Fisica, Universidade de Sao Paulo, \\ Rua do Matao Nr. 1371, Sao Paulo/SP, Brazil \\ E-mail: marisilvia.donadelli@cern.ch
}

With the end of Run 2, the LHC has delivered only $4 \%$ of the collision data expected to be available during its lifetime. The next data-taking campaign - Run 3 - will double the integrated luminosity the LHC accumulated in 10 years of operation. Run 3 will be the herald of the HL-LHC era, an era when $90 \%$ of total LHC integrated luminosity $\left(4 \mathrm{ab}^{-1}\right)$ will be accumulated allowing ATLAS to perform several precision measurements to constrain the Standard Model Theory (SM) in yet unexplored phase-spaces and in particular in the Higgs sector, only accessible at the LHC. Direct searches have so far provided no indication of new physics beyond the Standard Model, however, they can be complemented by indirect searches that allow extending the reach at higher scales. Indirect searches are based on the ability to perform very precise measurements, a highly complex task at a hadron collider that will require tight control of theoretical predictions, reconstruction techniques, and detector operation. Moreover, populating extreme regions of phase-space for multi-differential production cross-section analysis will require the development and validation of Monte Carlo phase-space biasing techniques and efficient integration methods to produce the billions of events needed to cope with higher luminosities. To answer the quest for high precision measurements in a high luminosity environment, a comprehensive upgrade of the detector and associated systems was devised and planned to be carried out in two phases. The Phase-I upgrade program foresees new features for the muon detector, for the electromagnetic calorimeter trigger system, and for all trigger and data acquisition chain and will operate to accumulate about 350 $\mathrm{fb}^{-1}$ of integrated luminosity during Run 3. The Phase-II upgrade comprises a completely new all-silicon tracker with extended rapidity coverage that will replace the current inner tracker detector; the calorimeters and muon systems will have their trigger and data acquisition systems fully redesigned, allowing the implementation of a free-running readout system. Finally, a new subsystem called High Granularity Timing Detector will aid the track-vertex association in the forward region by incorporating timing information into the reconstructed tracks.

The Ninth Annual Conference on Large Hadron Collider Physics - LHCP2021

7-12 June 2021

Online

\footnotetext{
${ }^{*}$ Speaker
} 
Physics motivations and detector upgrades for the new era of the ATLAS experimentMarisilvia Donadelli, on behalf of the ATLAS Collaboration

\section{Introduction}

The High-Luminosity Large Hadron Collider (HL-LHC) project at CERN [1] planned to start in mid-2027 aims to deliver a total of $4 \mathrm{ab}^{-1}$ of integrated luminosity after a 10-year period of operations. By then, the LHC will have hit all its design parameters: a center-of-mass collision energy of $\sqrt{s}=14 \mathrm{TeV}$ and an instantaneous luminosity of $7.5 \times 10^{34} \mathrm{~cm}^{-2} \mathrm{~s}^{-1}$, from which 200 simultaneous $p p$ interactions per bunch crossing $(\langle\mu\rangle=200)$ and an average pileup density of 1.8 vertices/mm with local variations are expected.

The ATLAS experiment [2] will be upgraded aiming to maximise the physics performance for precision measurements up to $14 \mathrm{TeV}$ in this challenging environment of high charged particle multiplicity and high radiation levels, keeping acceptable trigger rates with low $p_{T}$ threshold and pileup mitigation across $|\eta|<4.0$.

In order to satisfy these requirements, two extended periods without physics operation are planned prior to the HL-LHC operation during which upgrades will be made to the ATLAS experiment. Long Shutdown 2 (LS2) which began in 2019 makes the Phase-I upgrades, and Long Shutdown 3 (LS3) which is currently planned to last from 2025 until mid 2027 will be used for the extensive Phase-II upgrades, which will allow ATLAS to cope with the higher luminosities expected at the HL-LHC, and provide new capabilities.

The ATLAS Collaboration has planned a broad HLLHC physics program of precision measurements and searches for physics Beyond the Standard Model (BSM). This program includes measurements of the Higgs boson properties, Electroweak and QCD measurements, Flavour Physics studies, SUSY and Dark Matter BSM

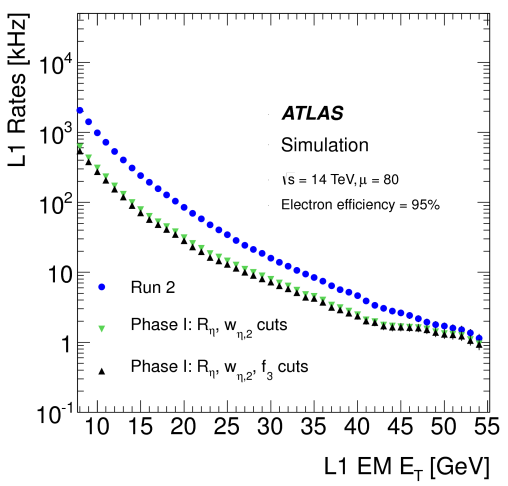

Figure 1: Level-1 trigger rates for a $95 \%$ electron efficiency as a function of the EM $E_{\mathrm{T}}$ threshold with $\langle\mu\rangle=80$ considering Run 2 (in blue) and the upgrade conditions (in green and black) [3].

\section{searches [4-6].}

This contribution is divided as follows. Section 2 will cover aspects of the Phase-I upgrade, and Section 3 will bring some of the new ATLAS detector Phase-II subsystems. Sections 4-5 will highlight some of the above mentioned studies that have been performed with either a full simulation of the ATLAS detector upgrades or with extrapolation from results obtained from Run 2 LHC data.

\section{LAr Calorimeter Trigger Phase-I Upgrade}

The Phase-I upgrade of the ATLAS LAr calorimeter will provide higher-granularity, higherresolution and longitudinal shower information from the calorimeter to the Level-1 trigger processors [3]. For Run 3, the so-called "Super Cells" will replace the coarse calorimeter trigger information based on the concept of a "Trigger Tower". This upgrade improves the trigger energy resolution and efficiency for selecting electrons, photons, $\tau$ leptons, jets, and missing transverse momentum $\left(E_{\mathrm{T}}^{\mathrm{miss}}\right)$, while enhancing discrimination against backgrounds and fakes in an environment with high pileup. The increased spatial information provided by the "Super Cells" allows use 
Physics motivations and detector upgrades for the new era of the ATLAS experiment Marisilvia Donadelli, on behalf of the ATLAS Collaboration

of shower shape discrimination at trigger level, as Fig. 1 demonstrates that for a given bandwidth the Level-1 threshold can be lowered with the proposed upgrade.

The Phase-I upgrade program also foresees new features for the muon detector, for all trigger and data acquisition chain and will operate to accumulate about $350 \mathrm{fb}^{-1}$ of integrated luminosity during Run 3.

\section{Inner Tracker and High-Granularity Timing Detector Phase-II Upgrades}

To cope with the challenging environment to particle detectors due to pileup at HL-LHC, the current Inner Detector of the ATLAS experiment will be replaced with a new all-silicon Inner Tracker (ITk) during the PhaseII upgrade. The ITk consists of a pixel subsystem with a pseudorapidity coverage of $|\eta|<4.0$ [8] and a strip subsystem covering the interval $|\eta|<2.7$ [9]. The new detector is sufficiently radiation tolerant (up to $9.9 \mathrm{MGy}$ ) with replacement of two inner layers after $2000 \mathrm{fb}^{-1}$ of accumulated luminosity. The project foresees improvements in geometry and materials, providing refined track

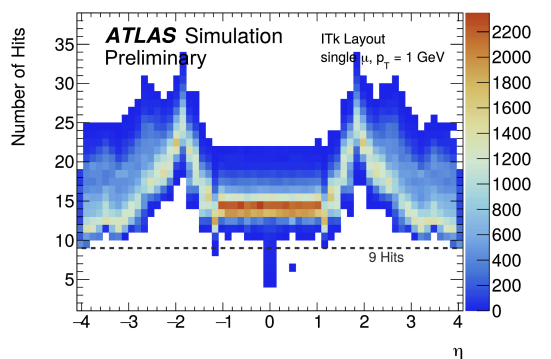

Figure 2: Hit coverage of the ITk Layout based on a sample of single muon events with $p_{\mathrm{T}}=1$ $\mathrm{GeV}$ [7]. measurement with a minimum number of 9 hits for full detector acceptance of $|\eta|<4.0$, as shown in Fig. 2.

The new High Granularity Timing Detector (HGTD),

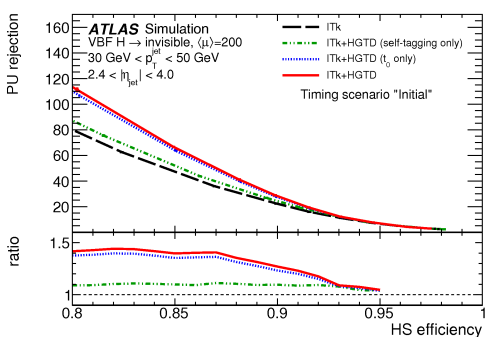

Figure 3: Pileup jet rejection as a function of hard-scatter jet efficiency in the $2.4<|\eta|<4.0$ region at the beginning of the lifetime of the detector, VBF $H \rightarrow$ invisible sample, for the ITk-only and combined ITk+HGTD reconstruction [10]. based on low gain avalanche detector technology [10], will cover the $2.4<|\eta|<4.0$ pseudorapidity region. The HGTD will aid the track-vertex association in the forward region by incorporating timing information (resolution of $30 \mathrm{ps}$ ) into the reconstructed tracks, complementing the ITk with pileup mitigation up to $|\eta|=4.0$, a very important region for VBF processes like $H \rightarrow$ invisible, as shown in Fig. 3. The HGTD is also capable of providing independent measurements of bunch-by-bunch luminosity via hit counts, a key contribution for precise measurements like the Higgs boson couplings that require $O(1 \%)$ accuracy and can be limited by the luminosity uncertainties.

Other Phase-II upgrades include the calorimeters and muon systems, that will have their trigger and data acquisition systems fully redesigned, allowing the implementation of a free-running readout system.

\section{Projection studies of the Higgs boson}

ATLAS expects to measure the Higgs boson production cross sections with precision between $2.4 \%$ and $7.7 \%$ [11], as shown in Fig. 4. These studies are obtained with an extrapolation of Run 
Physics motivations and detector upgrades for the new era of the ATLAS experimentMarisilvia Donadelli, on behalf of the ATLAS Collaboration

2 results, assuming the same detector performance as of the 2015-2016 data-taking period, with experimental and theory uncertainties reduced by a factor of 2 .

The measurement of the Higgs boson self coupling $\lambda_{H H H}$, expected to be experimentally assessed by the Higgs boson pair production $(H H)$ is one of the cornerstones of the HL-LHC program. ATLAS has studied $H H$ production at the HL-LHC with three decay channels: $b \bar{b} b \bar{b}, b \bar{b} \gamma \gamma$ and $b \bar{b} \tau^{+} \tau^{-}[12]$. Of these, the $b \bar{b} b \bar{b}$ and $b \bar{b} \tau^{+} \tau^{-}$studies have been performed from results obtained from Run 2 and extrapolated to HL-LHC. The $b \bar{b} \gamma \gamma$ analysis performed truth-level Monte Carlo studies using parametric functions to emulate the expected detector response. Combined results from the three final states achieve a $3.0 \sigma$ significance when all systematic uncertainties are included. When these results are further combined with similar $H H$ production studies from the

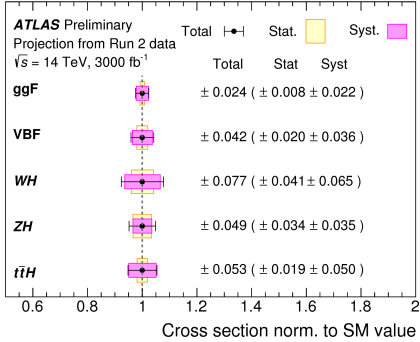

Figure 4: Expected result for the measured cross sections for the $g g \mathrm{~F}, \mathrm{VBF}, W H, Z H$ and $t \bar{t} H$ production modes normalised to their SM predictions assuming SM branching fractions [11]. CMS Collaboration, an overall significance of $4.0 \sigma$ can be reached with the HL-LHC dataset [5].

\section{Measurements of electroweak bosons}

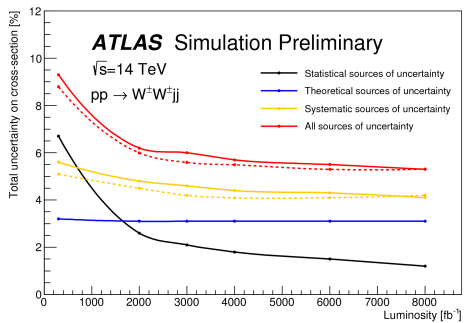

Figure 5: Projection of $W W j j$ uncertainties on the cross section as a function of integrated luminosity [13].
The study of vector boson scattering is an important goal of the HL-LHC physics program for it gives direct access to the nature of electroweak symmetry breaking mechanism. The main event signatures are $V V j j$ with leptonic decays of the bosons and therefore, detector upgrades that improve forward tracking and jet-finding capabilities are important for increasing the event selection acceptance. Prospects studies of $W W$ scattering cross section measurements with an expected total uncertainty of $6 \%$ of an integrated luminosity of $3000 \mathrm{fb}^{-1}$ are shown in Fig. 5. These studies were conducted with simulated events parameterised to take into account the expected detector effects in the high luminsosity environment. The same level of precision is expected for $W Z$ [14] scattering, while $Z Z$ scattering can be measured in the 1-8 $\sigma$ [15] range, depending on theory uncertainties.

Prospects studies of the $W$ boson mass and the electroweak mixing angle $\sin ^{2} \theta_{W}$ have been performed for the HL-LHC conditions. For the $W$ mass measurement, studies with a dedicated dataset collected at low instantaneous luminosity and moderate pileup, besides improved lepton acceptance at high $\eta$ suggest a statistical sensitivity on $m_{W}$ below $10 \mathrm{MeV}$ [16]. In the case of the $\sin ^{2} \theta_{W}$ measurement, through a study of the $\mathrm{Z}$ boson forward-backward asymmetry as a function of dielectron invariant mass and rapidity, both the improved electron acceptance and the large data sample result in substantial improvements indicating reachable LEP/SLD precision [17]. 
Physics motivations and detector upgrades for the new era of the ATLAS experimentMarisilvia Donadelli, on behalf of the ATLAS Collaboration

\section{References}

[1] O. Aberle et al., High-Luminosity Large Hadron Collider (HL-LHC): Technical design report. CERN Yellow Reports: Monographs. CERN, 2020. https: //cds . cern. ch/record/2749422.

[2] ATLAS Collaboration, JINST 3 (2008) S08003.

[3] ATLAS Liquid Argon Calorimeter Phase-I Upgrade: Technical Design Report, tech. rep., CERN, Sep, 2013. http://cds . cern . ch/record/1602230.

[4] R. K. Ellis et al., Physics Briefing Book: Input for the European Strategy for Particle Physics Update 2020. 10, 2019. arXiv: 1910.11775 [hep-ex].

[5] M. Cepeda et al., CERN Yellow Rep. Monogr. 7 (2019) 221-584, arXiv: 1902.00134 [hep-ph].

[6] P. Azzi et al., CERN Yellow Rep. Monogr. 7 (2019) 1-220, arXiv: 1902.04070 [hep-ph].

[7] Expected Tracking Performance of the ATLAS Inner Tracker at the HL-LHC, tech. rep., CERN, Mar, 2019. http : //cds . cern. ch/record/2669540.

[8] Technical Design Report for the ATLAS Inner Tracker Pixel Detector, tech. rep., CERN, Sep, 2017. http: //cds. cern. ch/record/2285585.

[9] Technical Design Report for the ATLAS Inner Tracker Strip Detector, tech. rep., CERN, Apr, 2017. http: //cds . cern . ch/record/2257755.

[10] Technical Design Report: A High-Granularity Timing Detector for the ATLAS Phase-II Upgrade, tech. rep., CERN, Jun, 2020. http : //cds . cern . ch/record/2719855.

[11] Projections for measurements of Higgs boson cross sections, branching ratios, coupling parameters and mass with the ATLAS detector at the HL-LHC, tech. rep., CERN, Dec, 2018. https: //cds . cern. ch/record/2652762.

[12] Measurement prospects of the pair production and self-coupling of the Higgs boson with the ATLAS experiment at the HL-LHC, tech. rep., CERN, Dec, 2018. http: //cds . cern. ch/record/2652727.

[13] Prospects for the measurement of the $W^{ \pm} W^{ \pm}$scattering cross section and extraction of the longitudinal scattering component in pp collisions at the High-Luminosity LHC with the ATLAS experiment, tech. rep., CERN, Dec, 2018. http : //cds . cern. ch/record/2652447.

[14] Prospective study of vector boson scattering in WZ fully leptonic final state at HL-LHC, tech. rep., CERN, Oct, 2018. http: //cds . cern. ch/record/2645271.

[15] Prospect study of electroweak production of a $Z$ boson pair plus two jets at the HL-LHC, tech. rep., CERN, Nov, 2018. https: //cds . cern. ch/record/2647219. 
[16] Prospects for the measurement of the W-boson mass at the HL-and HE-LHC, tech. rep., CERN, Oct, 2018. http: //cds . cern. ch/record/2645431.

[17] Prospect for a measurement of the Weak Mixing Angle in $p p \rightarrow Z / \gamma^{*} \rightarrow e^{+} e^{-}$events with the ATLAS detector at the High Luminosity Large Hadron Collider, tech. rep., CERN, Nov, 2018. http: //cds . cern. ch/record/2649330. 